\title{
NOTES
}

\section{On the Role of Electrostatic Interactions in the Enantioselective Recognition of Phenylalanine in Molecularly Imprinted Polymers Incorporating $\boldsymbol{\beta}$-Cyclodextrin}

\author{
Sergey A. PILETSKY, ${ }^{1}$ Håkan S. ANDERsson,${ }^{2}$ and Ian A. Nicholls ${ }^{2, \dagger}$ \\ ${ }^{1}$ Institute of BioScience and Technology, Cranfield University at Silsoe, Bedfordshire, MK45 4DT, UK \\ ${ }^{2}$ Department of Chemistry \& Biomedical Sciences, University of Kalmar, SE-391 82 Kalmar, Sweden
}

(Received May 17, 2005; Accepted June 22, 2005; Published October 15, 2005)

\begin{abstract}
KEY WORDS Molecular Imprinting / Template Polymerization / Enantioselectivity / Phenylalanine / $\beta$-Cyclodextrin / Acrylic Polymers /

[DOI 10.1295/polymj.37.793]
\end{abstract}

Molecular imprinting is a means for producing synthetic polymers with predetermined ligand selectivities. $^{1-4}$ The method relies upon the formation of complexes between functionalized monomers and a template molecule in the pre-polymerization solution. These complexes, if maintained during the polymerization process, will render a polymer possessing recognition sites with template-complementary functionality and topography. After extraction of the template, the polymer can be used for selective recognition of the template molecule from mixtures of closely related structures. In some instances, selectivities comparable with those of biological receptors and antibodies have been reported. ${ }^{5,6}$

Most molecular imprinting protocols rely mainly upon electrostatic interactions as the basis for prepolymerization template complexation. Although these protocols have proven very successful when applied in relatively non-polar polymerization mixtures, results have been somewhat modest when using template structures with little or no solubility in non-polar solvents, e.g. non-derivatized nucleotides, amino acids and peptides. Further developments, perhaps based upon the use of metal coordination or other multidentate-type ligands as the basis for pre-polymerization template complexation, ${ }^{7}$ are required in order to significantly improve polymer performance. As a promising alternative approach, several recent studies report the use of cyclodextrin (CD) derived structures as functional monomers for molecular imprinting in aqueous environments, where the hydrophobic binding pocket of the CD is utilized to recognize water soluble structures containing hydrophobic moieties. ${ }^{8-12}$ In work by our group, ${ }^{13}$ terpolymers selective for either of the enantiomers of phenylalanine (1) were developed using the functional monomers bisacryloyl $\beta$-CD (2) and 2-acryloylamido-2-methyl-1-propane sulfonic acid (AMPSA, 3), and the cross-linking agent $N, N^{\prime}-$ diacryloylpiperazine (DAP, 4), Figure 1. The roles of the hydrophobic interacting CD and the electrostatic interacting sulfonic acid monomers were examined with chromatographic analyses. In the present study, this system has been further explored through the synthesis of several novel polymer systems, where AMPSA, the electrostatic interacting functional monomer, has been successively replaced/complemented with 2-(dimetylamino)ethyl methacrylate (DEAEM, 5), urocanic acid ethyl ester (UAEE, 6), and 2-(trifluoromethyl)acrylic acid (TFMAA, 7).

\section{EXPERIMENTAL}

\section{General Information}

Chemicals and solvents were of analytical or HPLC grade and obtained from commercial sources. Monomers containing inhibitors were purified prior to use. Bisacryloyl $\beta$-cyclodextrin (2) was synthesised from $\beta$-cyclodextrin and acryloyl chloride as previously described. ${ }^{13}$

\section{Polymer Syntheses}

A series of molecularly imprinted and reference polymers was prepared, Table I, following a previously described procedure. ${ }^{13}$ Briefly, in a typical imprinted polymer preparation, the template, D-phenylalanine, was dissolved in water in a $50 \mathrm{~mL}$ screwcap borosilicate glass vial. The appropriate amounts of monomer(s) and cross-linker were added, and the tube was purged thoroughly with nitrogen before addition of the initiator system: $\left(\mathrm{NH}_{4}\right)_{2} \mathrm{~S}_{2} \mathrm{O}_{8}(200 \mu \mathrm{L}, 50 \%$ $\mathrm{w} / \mathrm{v})$ and TEMED $(50 \mu \mathrm{L})$. After sonication $(5 \mathrm{~s})$ of the reaction mixture, polymerization was carried

${ }^{\dagger}$ To whom correspondence should be addressed (Tel: +46-480-446258, Fax: +46-480-446244, E-mail: ian.nicholls@ hik.se). 


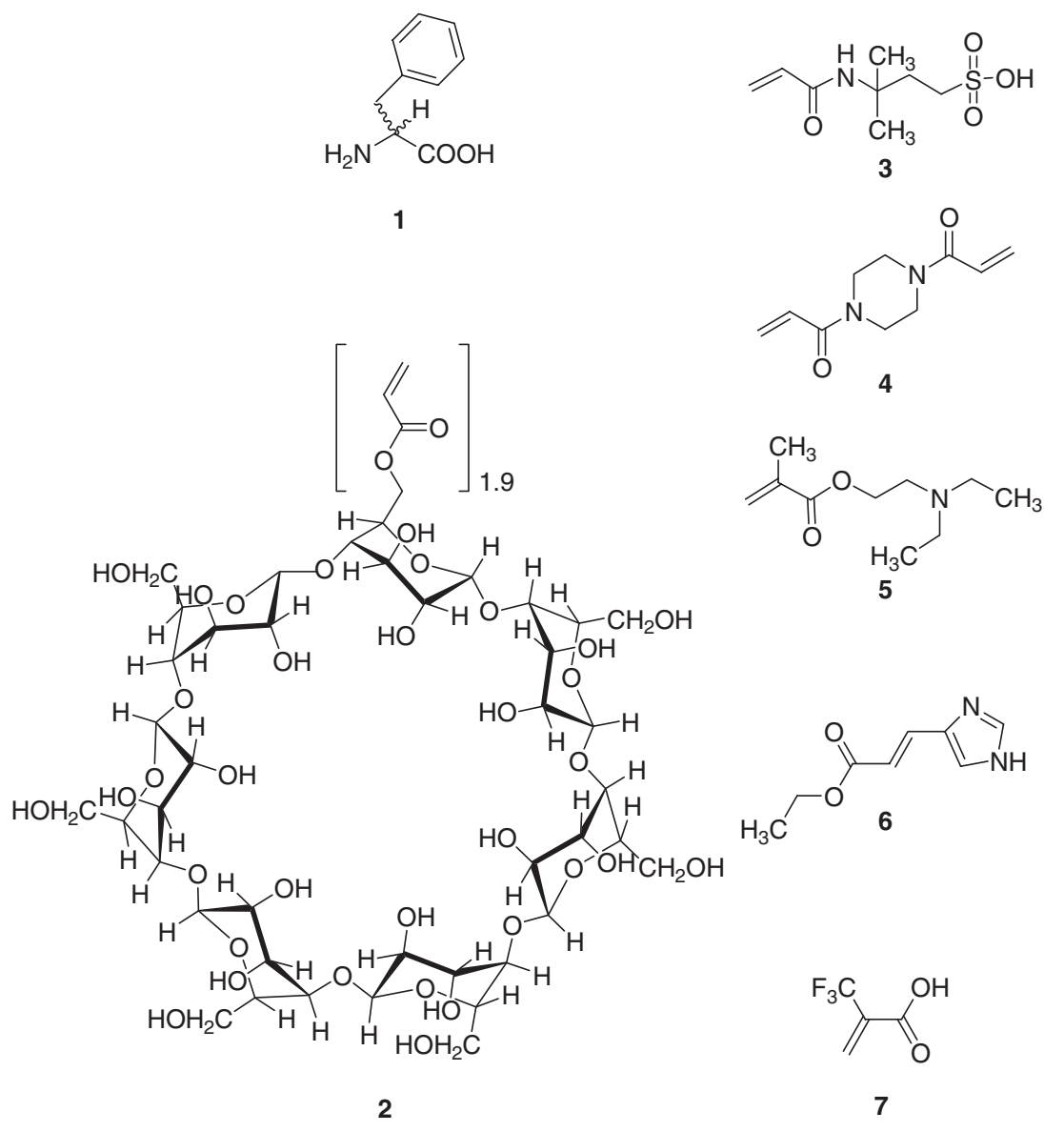

Figure 1. Phenylalanine (1), $\beta$-cyclodextrin monomer (2), 2-acryloylamido-2-methylpropane sulfonic acid (3), $N, N^{\prime}$-diacryloylpiperazine (4), 2-(diethylamino)ethyl methacrylate (5), urocanic acid ethyl ester (6), 2-(trifluoromethyl)acrylic acid (7).

Table I. Polymer compositions ${ }^{\mathrm{a}}$

\begin{tabular}{ccccccccc}
\hline Polymer & AMPSA $^{\mathrm{c}}$ & DEAEM $^{\mathrm{d}}$ & UAEE $^{\mathrm{e}}$ & TFMAA $^{\mathrm{f}}$ & CD $^{\mathrm{g}}$ & DAP $^{\mathrm{h}}$ & D-Phe & Water $^{\text {DF }}$ \\
\hline $\mathbf{P}(\mathbf{1})^{\mathrm{b}}$ & 0.8 & - & - & - & 0.4 & 16 & 0.4 & 200 \\
$\mathbf{P}(\mathbf{B})^{\mathrm{b}}$ & 0.8 & - & - & - & 0.4 & 16 & - & 200 \\
$\mathbf{P}(\mathbf{2})^{\mathrm{b}}$ & 0.8 & - & - & - & - & 16 & 0.4 & 200 \\
$\mathbf{P}(\mathbf{3})^{\mathrm{b}}$ & - & - & - & - & 0.4 & 16 & 0.4 & 200 \\
$\mathbf{P}(\mathbf{4})$ & 0.8 & - & 0.4 & - & 0.4 & 16 & 0.4 & 200 \\
$\mathbf{P}(\mathbf{5})$ & 0.8 & - & 0.8 & - & 0.4 & 16 & 0.4 & 200 \\
$\mathbf{P}(\mathbf{6})$ & - & 0.8 & - & - & 0.4 & 16 & 0.4 & 200 \\
$\mathbf{P}(7)$ & - & - & - & 0.8 & 0.4 & 16 & 0.4 & 200 \\
\hline
\end{tabular}

${ }^{a}$ All quantities are in mmol. ${ }^{b}$ Reported previously. ${ }^{13}$ c2-acryloylamido-2-methylpropane sulfonic acid. d2-(diethylamino)ethyl methacrylate. ${ }^{\mathrm{e}}$ urocanic acid ethyl ester. ${ }^{\mathrm{f}}$ 2-(trifluoromethyl)acrylic acid. ${ }^{\mathrm{g}} \beta$-cyclodextrin monomer. ${ }^{\mathrm{h}} N, N^{\prime}$-diacryloylpiperazine.

out at $75^{\circ} \mathrm{C}$ for $18 \mathrm{~h}$. The resultant polymer was crushed and ground and particles were collected by sedimentation and filtration through sieves $(63 \mu \mathrm{m}$ and $25 \mu \mathrm{m})$ with water. The polymers were washed with water $(100 \mathrm{~mL} \times 10)$ to remove unreacted monomers and residual template. Finally, the polymer suspension (25-63 $\mu \mathrm{m})$ was used for packing of HPLC columns. The reference polymer $\mathbf{P}(\mathbf{B})$ was similarly prepared and processed, though in the absence of template.

\section{Polymer Physical Characterisation}

Combustion analyses were performed by Mikrokemi AB (Uppsala, Sweden). Surface area analysis was performed on a Micrometrics Flowsorb II 2300 instrument, using $30 \% \mathrm{~N}_{2}$ in Ar. P(1): C 56.5, $\mathrm{H} 7.5$, N 12.8, S 0.7\%. BET surface area, $362 \mathrm{~m}^{2} / \mathrm{g}$; micropore area, $42.4 \mathrm{~m}^{2} / \mathrm{g}$, micropore volume, 0.014 $\mathrm{cm}^{3} / \mathrm{g}$; average pore diameter, $80.2 \AA$. $\mathbf{P}(\mathbf{B}): \mathrm{C} 56.1$, $\mathrm{H} 7.5, \mathrm{~N} 12.8$, S $0.7 \%$. BET surface area, $324 \mathrm{~m}^{2} / \mathrm{g}$; micropore area, $37.3 \mathrm{~m}^{2} / \mathrm{g}$, micropore volume, 0.013 
$\mathrm{cm}^{3} / \mathrm{g}$; average pore diameter, $80.0 \AA$ A. P(2): C 54.2, H 7.7, N 12.4, S $0.7 \%$. BET surface area, $454 \mathrm{~m}^{2} / \mathrm{g}$; micropore area, $111.5 \mathrm{~m}^{2} / \mathrm{g}$, micropore volume, 0.046 $\mathrm{cm}^{3} / \mathrm{g}$; average pore diameter, $80.3 \AA$ A. $\mathbf{P}(\mathbf{3})$ : C $58.4, \mathrm{H}$ 7.6, N $13.4 \%$. BET surface area, $321 \mathrm{~m}^{2} / \mathrm{g}$; micropore area, $42.9 \mathrm{~m}^{2} / \mathrm{g}$, micropore volume, $0.015 \mathrm{~cm}^{3} / \mathrm{g}$; average pore diameter, $64.9 \AA$. $\mathbf{P ( 4 ) : ~ C ~} 56.4, \mathrm{H} 7.5$, $\mathrm{N} 13.0, \mathrm{~S} 0.7 \%$. BET surface area, $310 \mathrm{~m}^{2} / \mathrm{g}$; micropore area, $39.8 \mathrm{~m}^{2} / \mathrm{g}$, micropore volume, $0.014 \mathrm{~cm}^{3} /$ $\mathrm{g}$; average pore diameter, $77.6 \AA$. P(5): C 59.9, H 7.7, N 13.8, S $0.4 \%$. BET surface area, $251 \mathrm{~m}^{2} / \mathrm{g}$; micropore area, $30.3 \mathrm{~m}^{2} / \mathrm{g}$, micropore volume, 0.010 $\mathrm{cm}^{3} / \mathrm{g}$; average pore diameter, $77.8 \AA$. $\mathbf{P ( 6 )}$ : C 59.2, $\mathrm{H} 7.8$, N $13.4 \%$. BET surface area, $240 \mathrm{~m}^{2} / \mathrm{g}$; micropore area, $35.0 \mathrm{~m}^{2} / \mathrm{g}$, micropore volume, $0.013 \mathrm{~cm}^{3} /$ $\mathrm{g}$; average pore diameter, $75.8 \AA$. $\mathbf{P ( 7 )}$ : C $57.5, \mathrm{H}$ $7.1, \mathrm{~N} 12.7 \%$. BET surface area, $232 \mathrm{~m}^{2} / \mathrm{g}$; micropore area, $25.0 \mathrm{~m}^{2} / \mathrm{g}$, micropore volume, $0.008 \mathrm{~cm}^{3} / \mathrm{g}$; average pore diameter, $105.7 \AA$.

\section{High Performance Liquid Chromatography}

Polymer particles were packed into stainless steel HPLC columns $(100 \times 4.6 \mathrm{~mm}$ i.d.), using an air driven fluid pump (Haskel Engineering Supply Co.). Columns were packed at 340 bar using water. Polymers were washed on-line with water until a stable base line was obtained. Chromatographic experiments were carried out at $25^{\circ} \mathrm{C}$ using an HPLC system comprising a thermostatted column oven (Croco-cil, C.I.L. France), a Series 200 LC Pump (Perkin Elmer, Norwalk, CT, USA) and a 785A Programmable absorbance detector (Applied Biosystems, Roissy, France). Samples containing D-Phe or L-Phe $(1 \mathrm{mg} / \mathrm{mL})$ were prepared in the eluent and $50 \mu \mathrm{L}$ of solution were injected for analysis. Analyses were run at a flow rate of $0.4 \mathrm{~mL} / \mathrm{min}$ with detection at $257 \mathrm{~nm}$, using water/ acetonitrile $(3 / 7, \mathrm{v} / \mathrm{v})$ as the mobile phase. Optimization of the mobile phase was reported in previous work. ${ }^{13}$ All reported chromatographic data represent the results of at least 4 concordant experiments. Capacity factors $\left(k^{\prime}\right)$ were determined from $k^{\prime}=(V-$ $\left.V_{\mathrm{o}}\right) / V_{\mathrm{o}}$, where $V$ is the retention volume of a given species and $V_{\mathrm{o}}$ is the retention volume of the void marker (acetone). Effective enantioseparation factors $(\alpha)$ were calculated from the relationship $\alpha=k_{\mathrm{D}}^{\prime} /$ $k_{\mathrm{L}}^{\prime}$, where $k_{\mathrm{D}}^{\prime}$ and $k_{\mathrm{L}}^{\prime}$ are the capacity factors of the D- and L-Phe, respectively.

\section{RESULTS AND DISCUSSION}

Functional monomer-template recognition was envisaged to arise from a combination of binding of the template phenyl moiety into the hydrophobic cavity of the cyclodextrin, ion pairing, and hydrogen bonding of the amine and carboxyl to the electrostatic interacting monomer, in conjunction with van der Waals shape complementarity. The electrostatic functional monomers used in this study range from the strongly acidic AMPSA (sulfonic acid) to the strongly basic DEAEM. The use of combinations of functionally different monomers was expected to allow the development of synthetic polymers better capable of mimicking protein systems, which themselves are constructed with a diverse array of functionally different monomers, the amino acids.

The enantioselectivity of D- and L-phenylalanine imprinted CD/AMPSA/DAP polymers has previously been studied using chromatographic techniques. ${ }^{8,13}$ The contribution of the imprinting process to polymer selectivity is sufficient to surmount the inherent selectivity of the cyclodextrin for the L-enantiomer, as illustrated upon comparison of the imprinted and non-imprinted AMPSA based polymers $\mathbf{P}(\mathbf{1})$ and $\mathbf{P}(\mathbf{B})$. Optimal ligand selectivity was demonstrated when using an eluent of intermediate polarity (acetonitrile/water, 7:3), which illustrated the significance of both hydrophobic and electrostatic interactions for ligand recognition, and was employed for evaluation of the present polymer systems (Table II).

The significance of a combination of both electrostatic and hydrophobic contributions to template recognition is further highlighted by the absence of recognition when $\mathrm{CD}$ is omitted from the protocol $(\mathbf{P}(\mathbf{2}))$. Furthermore, in $\mathbf{P ( 3 )}$, where AMPSA has been omitted from the protocol, the sole use of the neutral amide functionalities from the cross-linker provides an inadequate source of electrostatic interactions for D-phenylalanine recognition. Substitution of the highly acidic functional monomer AMPSA for the basic amine containing DEAEM $(\mathbf{P}(\mathbf{6}))$ also resulted in a complete loss of template selectivity. The unfavorable effect of this monomer combination is also reflected in the very low capacity factors obtained. Attempts to improve recognition through the addition of extra functionality via introduction of UAEE, $\mathbf{P}(4)$ and

Table II. Recognition of D- and L-Phe by polymeric stationary phases

\begin{tabular}{cccc}
\hline Polymer & $k_{\mathrm{D}}^{\prime}$ & $k_{\mathrm{L}}^{\prime}$ & $\alpha$ \\
\hline $\mathbf{P}(\mathbf{1})$ & 1.07 & 0.97 & 1.10 \\
$\mathbf{P}(\mathbf{B})$ & 0.98 & 1.00 & 0.98 \\
$\mathbf{P}(\mathbf{2})$ & 1.02 & 1.02 & 1.00 \\
$\mathbf{P}(\mathbf{3})$ & 1.10 & 1.12 & 0.98 \\
$\mathbf{P}(\mathbf{4})$ & 0.71 & 0.70 & 1.01 \\
$\mathbf{P ( 5 )}$ & 0.60 & 0.60 & 1.00 \\
$\mathbf{P}(\mathbf{6})$ & 0.53 & 0.54 & 0.98 \\
$\mathbf{P}(\mathbf{7})$ & 0.96 & 0.81 & 1.19 \\
\hline
\end{tabular}

A sample of $0.1 \mathrm{mg} / \mathrm{mL}$ samples of pure phenylalanine enantiomer was analyzed during each run. Eluent: $\mathrm{H}_{2} \mathrm{O}$ /acetonitrile $(30: 70, v / v)$. Standard deviations were below $5 \%$ for all $k^{\prime}$. 


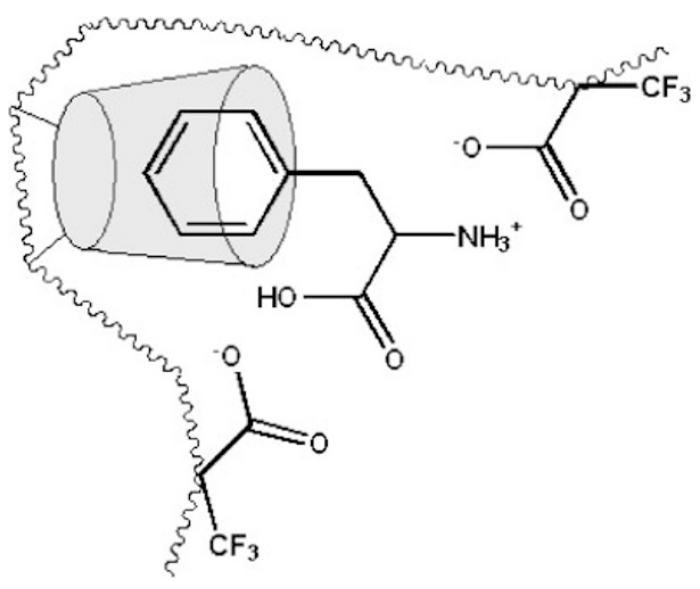

Figure 2. Schematic illustration of the $\mathbf{P}(7)$ phenylalanine recognition site.

$\mathbf{P}(\mathbf{5})$, actually has an adverse effect on recognition relative to $\mathbf{P}(\mathbf{1})$. However, substitution of AMPSA for the more weakly acidic TFMAA, $\mathbf{P}(7)$, demonstrated an enhancement of template enantioselectivity $(\alpha=$ 1.19) relative to the other systems studied, which corresponds to a $5.7 \mathrm{~kJ} \mathrm{~mol}^{-1}$ increase in the relative strength of enantiomer binding as compared to that previously observed with $\mathbf{P}(\mathbf{1})(\alpha=1.10)$. A likely explanation for this effect is the more rigid structure of TFMAA relative to AMPSA. The utility of TFMAA as a functional monomer in organic solvent based molecular imprinting protocols has previously been extensively studied by the group of Takeuchi. ${ }^{14}$ A schematic illustration of the $\mathbf{P}(7)$ recognition site is presented in Figure 2.

\section{CONCLUSIONS}

In this study, a series of molecularly imprinted polymers have been prepared in water. Acrylated $\beta$ cyclodextrin was employed as a hydrophobic monomer while four electrostatically interacting monomers were used successively, by analogy to site-directed mutagenesis, for the imprinting of D-phenylalanine. The most highly selective polymer system, employing 2-(trifluoromethacrylic) acid as a co-monomer, exhibited an enantioseparation factor $(\alpha)$ of 1.19 when using the polymer as an HPLC stationary phase. This represents an improvement over previously reported systems.

Acknowledgment. This work was supported by the Swedish Research Council (VR), Carl-Tryggers Foundation, Swedish Knowledge Foundation (KKS), Graninge Foundation and the University of Kalmar. The authors also wish to express their gratitude to Dr. Jesper G. Karlsson for comments on the manuscript.

\section{REFERENCES}

1. K. J. Shea, M. Yan, and O. Ramström, Ed., Molecularly Imprinted Materials-Sensors and Other Devices, Mater. Res. Soc. Symp. Proc., 723, 2002.

2. S. A. Piletsky and A. Turner, Ed., "Molecular Imprinting of Polymers," Landes Biosciences, Georgetown USA, 2004.

3. M. Komiyama, T. Takeuchi, T. Mukawa, and H. Asanuma, "Molecular Imprinting-from Fundamentals to Applications," Wiley-VCH, Weinheim, Germany, 2003.

4. B. Sellergren, Ed., In "Molecularly Imprinted Polymers, Man Made Mimics of Antibodies and their Application in Analytical Chemistry," Elsevier, Amsterdam, 2001.

5. G. Vlatakis, L. I. Andersson, R. Müller, and K. Mosbach, Nature, 361, 645 (1993).

6. N. Lavignac, C. J. Allender, and K. R. Brain, Anal. Chim. Acta, 510, 139 (2004).

7. S. Striegler, J. Chromatogr. B, 804, 183 (2004).

8. S. A. Piletsky, H. S. Andersson, and I. A. Nicholls, J. Mol. Recognit., 11, 83 (1998).

9. K. Sreenivasan, J. Appl. Polym. Sci., 70, 15 (1998).

10. H. Asanuma, R. Kakazu, M. Shibata, T. Hishiya, and M. Komiyama, Supramol. Sci., 5, 417 (1998).

11. H. Kitano and Y. Taira, Langmuir, 18, 5835 (2002).

12. K. Chmurski, A. Temeriusz, and R. Bilewicz, Anal. Chem., 75, 5687 (2003).

13. S. A. Piletsky, H. S. Andersson, and I. A. Nicholls, Macromolecules, 32, 633 (1999).

14. J. Matsui, O. Doblhoff-Dier, and T. Takeuchi, Anal. Chim. Acta, 343, 1 (1997). 